PROCEEDINGS OF THE

AMERICAN MATHEMATICAL SOCIETY

Volume 133, Number 11, Pages 3371-3379

S 0002-9939(05)08073-1

Article electronically published on June 20, 2005

\title{
THE SHARP HAUSDORFF MEASURE CONDITION FOR LENGTH OF PROJECTIONS
}

\author{
YUVAL PERES AND BORIS SOLOMYAK \\ (Communicated by David Preiss)
}

\begin{abstract}
In a recent paper, Pertti Mattila asked which gauge functions $\varphi$ have the property that for any Borel set $A \subset \mathbb{R}^{2}$ with Hausdorff measure $\mathcal{H}^{\varphi}(A)>0$, the projection of $A$ to almost every line has positive length. We show that finiteness of $\int_{0}^{1} \frac{\varphi(r)}{r^{2}} d r$, which is known to be sufficient for this property, is also necessary for regularly varying $\varphi$. Our proof is based on a random construction adapted to the gauge function.
\end{abstract}

\section{INTRODUCTION}

A classical theorem of Marstrand [4] states that if a planar Borel set $A$ has Hausdorff dimension strictly greater than 1 , then the orthogonal projection of $A$ to almost every line has positive length. If $A$ has dimension 1, the situation is more delicate. Recall that given a positive function $\varphi$ on $(0, \infty)$, the Hausdorff measure $\mathcal{H}^{\varphi}$ is defined as $\mathcal{H}^{\varphi}(A)=\lim _{\epsilon \downarrow 0} \inf \left\{\sum_{i} \varphi\left(\operatorname{diam} A_{i}\right): A \subset \bigcup_{i} A_{i}, \operatorname{diam} A_{i}<\epsilon\right\}$.

In his definitive survey on Hausdorff dimension and projections, Mattila [6] asked which gauge functions $\varphi$ have the property that for any Borel set $A \subset \mathbb{R}^{2}$ with $\mathcal{H}^{\varphi}(A)>0$, the projection of $A$ to almost every line has positive length. In this paper we settle Mattila's question, showing that an integral condition known to be sufficient for this property is also necessary; a partial result in this direction was obtained in [2]. The solution also clarifies the relation between Hausdorff measures and integral-geometric measure.

Notation. Let $\eta$ be the isometry-invariant measure on the space of all lines in $\mathbb{R}^{2}$, and define the integral-geometric measure $\mathcal{I}^{1}$ on Borel sets in $\mathbb{R}^{2}$ by $\mathcal{I}^{1}(A)=$ $\int \#(A \cap \ell) d \eta(\ell)$. (See (1.4) and [5], Section 5.14.) Let $p_{\theta}$ denote the orthogonal projection from $\mathbb{R}^{2}$ onto the line through the origin making angle $\theta$ with the horizontal axis. We denote by $\mathcal{L}^{m}$ the $m$-dimensional Lebesgue measure. We write $f \asymp g$ if $f \leq$ const $\cdot g$ and $g \leq$ const $\cdot f$ for some uniform constant.

Theorem 1.1. Let $\varphi:(0, \infty) \rightarrow(0, \infty)$ be a (weakly) increasing function such that

$$
\varphi(r) / r^{2} \text { is (weakly) decreasing. }
$$

Received by the editors June 29, 2004.

2000 Mathematics Subject Classification. Primary 28A80; Secondary 28A75, 60D05, 28A78.

The research of the first author was partially supported by NSF grants \#DMS-0104073 and \#DMS-0244479. Part of this work was done while he was visiting Microsoft Research. The research of the second author was supported in part by NSF grant \#DMS-0099814. 
Then the following are equivalent:

(i) $\int_{0}^{1} \frac{\varphi(r)}{r^{2}} d r<\infty$.

(ii) If a Borel set $A \subset \mathbb{R}^{2}$ satisfies $\mathcal{H}^{\varphi}(A)>0$, then

$$
\mathcal{L}^{1}\left(p_{\theta}(A)\right)>0 \text { for almost all } \theta \in[0, \pi) .
$$

(iii) $\mathcal{H}^{\varphi}$ is absolutely continuous to $\mathcal{I}^{1}$ on Borel sets in $\mathbb{R}^{2}$.

The implication (i) $\Rightarrow$ (ii) is known: Indeed, (i) gives $\int_{0}^{1} \frac{1}{r} d \varphi(r)<\infty$ using integration by parts. By [1], Theorem IV.1, this implies that for sets $A$ with $\mathcal{H}^{\varphi}(A)>0$, the one-dimensional capacity of $A$ is positive; a theorem of Kaufman [3] (see also [5], Cor. 9.8) then yields (1.2). Clearly (ii) $\Rightarrow$ (iii) by definition. We establish the new implication (iii) $\Rightarrow$ (i) by a random construction; it is intriguing that so far, deterministic constructions yield less sharp results. See Corollary 1.3 below for an extension of Theorem 1.1 to higher dimensions. Note that for a Borel set $A \subset \mathbb{R}^{2}$, having positive one-dimensional capacity is not necessary for (1.2); rectifiable curves have zero capacity but certainly satisfy (1.2).

What if the gauge function does not satisfy the regularity condition (1.1)? In the following lemma we show that if $\varphi$ is increasing, then we can find another gauge function $\varphi_{1}$, so that $\varphi_{1}(r) / r^{2} \downarrow$ and $\mathcal{H}^{\varphi_{1}} \asymp \mathcal{H}^{\varphi}$. (Note, however, that it could happen that $\int_{0}^{1} \frac{\varphi(r)}{r^{2}} d r=+\infty$, but $\int_{0}^{1} \frac{\varphi_{1}(r)}{r^{2}} d r<\infty$; in that case, for $A$ with $\mathcal{H}^{\varphi}(A)>0$, almost every projection has positive length.)

Lemma 1.2. Suppose that $\varphi:(0, \infty) \rightarrow(0, \infty)$ is weakly increasing. Then there exists a weakly increasing $\varphi_{1}:(0, \infty) \rightarrow(0, \infty)$ such that $\varphi_{1}(r) / r^{2} \downarrow$, and

$$
\mathcal{H}^{\varphi_{1}}(A) \leq \mathcal{H}^{\varphi}(A) \leq C \cdot \mathcal{H}^{\varphi_{1}}(A)
$$

for all Borel sets $A \subset \mathbb{R}^{2}$.

The proof of the lemma will be presented at the end of the paper.

Remark. Joyce and Mörters [2] constructed a set $A \subset \mathbb{R}^{2}$ such that $0<\mathcal{H}^{\phi}(A)<\infty$, for some $\phi$ satisfying $\int_{0}^{1} \frac{\phi(r)^{\alpha}}{r^{1+\alpha}} d r<\infty$ for every $\alpha>1$, such that $\mathcal{L}^{1}\left(p_{\theta}(A)\right)=0$ for all $\theta$.

Next, we discuss higher dimensions. Let $\mathcal{H}^{m}$ denote $m$-dimensional Hausdorff measure in $\mathbb{R}^{n}$. Recall from [5], Section 5.14, that the integral-geometric measure $\mathcal{I}^{m}$ is defined on Borel sets $A \subset \mathbb{R}^{n}$ by

$$
\mathcal{I}^{m}(A)=\iint \#\left(A \cap p_{V}^{-1}(a)\right) d \mathcal{H}^{m}(a) d \gamma_{n, m}(V),
$$

where $p_{V}$ is the orthogonal projection to $V$, and $\gamma_{n, m}$ is the isometry-invariant measure on the Grassman manifold of $m$-dimensional subspaces of $\mathbb{R}^{n}$. The next corollary generalizes Theorem 1.1

Corollary 1.3. Let $m<n$ and suppose $\psi$ is a positive function on $(0, \infty)$ such that $\psi(r) / r^{m-1}$ is weakly increasing and $\psi(r) / r^{m+1}$ is weakly decreasing (these regularity conditions could be relaxed). Then the following are equivalent:

(i) $\int_{0}^{1} \frac{\psi(r)}{r^{m+1}} d r<\infty$.

(ii) If a Borel set $A \subset \mathbb{R}^{n}$ satisfies $\mathcal{H}^{\psi}(A)>0$, then $\mathcal{L}^{m}\left(p_{V}(A)\right)>0$ for $\gamma_{n, m}$ almost all $m$-dimensional subspaces $V \subset \mathbb{R}^{n}$.

(iii) $\mathcal{H}^{\psi}$ is absolutely continuous to $\mathcal{I}^{m}$ on Borel sets in $\mathbb{R}^{n}$. 
The implication (i) $\Rightarrow$ (ii) is known: it follows from combining [1], Theorem IV.1, with [5], Cor. 9.8. Since (ii) $\Rightarrow$ (iii) is obvious, we will only need to prove (iii) $\Rightarrow($ i), and this will follow from the same construction we use to establish the corresponding implication in Theorem 1.1 .

\section{Outline of the COnstruction}

To prove the implication (iii) $\Rightarrow$ (i) in Theorem 1.1 we establish the following.

Proposition 2.1. Let $\varphi$ be a weakly increasing function that satisfies (1.1) and $\int_{0}^{1} \frac{\varphi(r)}{r^{2}} d r=\infty$. Then there exists a Borel set $A \subset \mathbb{R}^{2}$ such that $\mathcal{H}^{\varphi}(A)>0$ and $\mathcal{L}^{1}\left(p_{\theta}(A)\right)=0$ for almost all $\theta \in[0, \pi)$.

The set $A$ is constructed as a random Cantor set in the plane; we will show that it has all the desired properties almost surely. This set is a (substantial) modification of a stochastically self-similar set constructed in [7].

Denote by $\mathcal{G}_{k}$ the collection of $4^{k}$ (closed) dyadic subsquares of the unit square $[0,1]^{2}$ having side length $2^{-k}$. We consider all dyadic subsquares as a rooted tree, with $[0,1]^{2}$ being the root and $\mathcal{G}_{k}$ being the set of nodes at the $k$ th level. For each node there are four edges leading to nodes at the next level (its "offspring").

First we inductively define random subsets $\mathcal{F}_{k} \subset \mathcal{G}_{k}$ for $k \geq 0$, and let $\mathcal{R}_{k}:=$ $\bigcup\left\{Q: Q \in \mathcal{F}_{k}\right\}$. We start with $\mathcal{F}_{0}=\mathcal{G}_{0}=\left\{[0,1]^{2}\right\}$. Passing from $\mathcal{F}_{k}$ to $\mathcal{F}_{k+1}$ is either deterministic or random, depending on $k$. Given $\mathcal{F}_{k}$, a set of dyadic squares of side length $2^{-k}$, we partition each of them into four dyadic subsquares of side length $2^{-k-1}$. Now, if the step is deterministic, then we keep all the squares, so that $\# \mathcal{F}_{k+1}=4 \# \mathcal{F}_{k}$ and $\mathcal{R}_{k+1}=\mathcal{R}_{k}$. If the step is random, then we choose one of the four subsquares uniformly at random, all of these choices being independent. This way we obtain a subset $\mathcal{F}_{k+1}$ with the same cardinality as $\mathcal{F}_{k}$. The gauge function $\varphi$ dictates whether we make a deterministic or random step. If we do $\alpha_{k}$ deterministic steps for $i<k$, then $\# \mathcal{F}_{k}=4^{\alpha_{k}}$, and we make sure that $4^{-\alpha_{k}} \asymp \varphi\left(2^{-k}\right)$. Once $\mathcal{F}_{k}$ and $\mathcal{R}_{k}$ are defined, we consider $\mathcal{R}=\bigcap_{k=1}^{\infty} \mathcal{R}_{k}$, which satisfies $0<\mathcal{H}^{\varphi}(\mathcal{R})<\infty$.

We do not know whether the resulting random set has a.e. projection of zero length (almost surely), so we modify the construction slightly, removing certain squares at specific levels chosen in advance. This yields a new random set $\mathcal{R}^{\prime}$ which has all the desired properties.

\section{Construction in detail}

Consider a gauge function $\varphi(r)$ as in Theorem 1.1. Without loss of generality, we can assume that $\varphi(1)=1$. Denoting by $\lfloor\cdot\rfloor$ the integer part, let

$$
\alpha_{n}=\left\lfloor\log \left(\varphi\left(2^{-n}\right)\right) / \log (1 / 4)\right\rfloor \text { for } n \geq 0,
$$

so that $\alpha_{0}=0$ and

$$
\varphi\left(2^{-n}\right) \leq 4^{-\alpha_{n}} \leq 4 \cdot \varphi\left(2^{-n}\right) .
$$

Since $\varphi(r)$ is increasing, we have $\alpha_{n} \geq \alpha_{n-1}$, and since $\varphi(r) / r^{2}$ is decreasing, we have $\alpha_{n} \leq \alpha_{n-1}+1$. Now,

$$
\infty=\int_{0}^{1} \frac{\varphi(r)}{r^{2}} d r=\sum_{n=0}^{\infty} \int_{2^{-n-1}}^{2^{-n}} \frac{\varphi(r)}{r^{2}} d r \leq \sum_{n=0}^{\infty} 2^{n+1} \varphi\left(2^{-n}\right) \leq \sum_{n=0}^{\infty} 2^{n+1} 4^{-\alpha_{n}},
$$




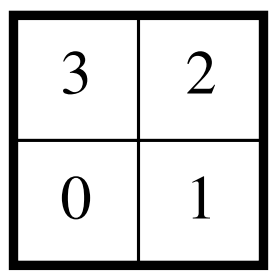

FiguRE 1. Labeling subsquares.

so

$$
\sum_{n=0}^{\infty} \lambda_{n}=\infty, \quad \text { where } \lambda_{n}:=2^{n} 4^{-\alpha_{n}} .
$$

Now we specify when we perform deterministic steps. The transition from $\mathcal{F}_{n-1}$ to $\mathcal{F}_{n}$ is deterministic if $\alpha_{n}>\alpha_{n-1}$, that is, $\alpha_{n}=\alpha_{n-1}+1$. Otherwise, the transition is random, as described in Section 2. This is well defined, and we make $\alpha_{n}$ deterministic steps on levels $i<n$, so $\# \mathcal{F}_{n}=4^{\alpha_{n}}$. Recall that $\mathcal{R}_{n}=\bigcup_{B \in \mathcal{F}_{n}} B$ and $\mathcal{R}=\bigcap_{n=1}^{\infty} \mathcal{R}_{n}$. Consider the probability measure $\mu$ on $\mathcal{R}$ defined as the weak* limit of uniform measures on $\mathcal{R}_{n}$. We claim that there exists $C_{1}>0$ such that

$$
\mu\left(B_{r}\right) \leq C_{1} \cdot \varphi(r)
$$

for any ball of radius $r$. Indeed, up to a multiplicative constant, we can replace the ball $B_{r}$ in (3.3) by a square $B \in \mathcal{F}_{n}$ where $2^{-n} \asymp r$. We have $\mu(B)=1 / \# \mathcal{F}_{n}=$ $4^{-\alpha_{n}} \asymp \varphi\left(2^{-n}\right)$, and the desired estimate follows. Thus, $\mathcal{H}^{\varphi}(\mathcal{R})>0$ by the Mass Distribution Principle. We can assume that $\lambda_{n}$ are bounded,

$$
\lambda_{n}<C_{2},
$$

for all $n$. Indeed, the set $\mathcal{R}_{n}$ consists of $4^{\alpha_{n}}$ squares of side length $2^{-n}$. If (3.4) does not hold, then $\inf \left(4^{\alpha_{n}} 2^{-n}\right)=0$, hence $\mathcal{H}^{1}(\mathcal{R})=0$, and then all projections of $\mathcal{R}$ have zero length.

Next we specify how we modify the set $\mathcal{R}$ by removing certain squares at prescribed levels. We label the four dyadic subsquares of a square as in Figure 1. This labeling induces a natural addressing scheme for each dyadic square $B \in \mathcal{G}_{k}$. The address has length $k$ and the symbols are from $\{0,1,2,3\}$; we write it as $\omega(B)=\left\{\omega_{i}(B)\right\}_{i=1}^{k}$.

For $B \in \mathcal{G}_{n}$ and $1 \leq i \leq n$ define

$$
Y_{i}(B)= \begin{cases}\lambda_{i}, & \text { if } \omega_{i}(B) \in\{0,2\} \\ 0, & \text { if } \omega_{i}(B) \in\{1,3\}\end{cases}
$$

We can consider $\mathcal{G}_{n}$ as a discrete probability space with the uniform measure. Then $\left\{Y_{i}\right\}_{i=1}^{n}$ are independent random variables, such that $\mathbf{P}\left(Y_{i}=\lambda_{i}\right)=\mathbf{P}\left(Y_{i}=0\right)=\frac{1}{2}$. Let

$$
S_{n}(B)=\sum_{i=1}^{n} Y_{k_{i}}(B)
$$

where $k_{i}$ is the $i$ th deterministic step, so that $\alpha_{k_{i}-1}=i-1$ and $\alpha_{k_{i}}=i$. Denote

$$
\gamma_{i}:=\lambda_{k_{i}}=2^{k_{i}} 4^{-\alpha_{k_{i}}}=2^{k_{i}} 4^{-i}
$$


then $\mathbf{E}\left(S_{n}\right)=\frac{1}{2} \sum_{i=1}^{n} \gamma_{i}$ and $\operatorname{Var}\left(S_{n}\right)=\frac{1}{4} \sum_{i=1}^{n} \gamma_{i}^{2}$. Observe that $S_{n}(B)$ depends only on the digits of $B$ corresponding to deterministic steps, hence it is independent of the event $B \in \mathcal{F}_{k_{n}}$ which can be considered on the same probability space $\mathcal{G}_{k_{n}}$.

We say that $B \in \mathcal{G}_{k_{n}}$ is deviant if

$$
\left|S_{n}(B)-\mathbf{E}\left(S_{n}\right)\right|>\mathbf{E}\left(S_{n}\right) / 2 .
$$

Using Chebyshev's inequality we obtain

$$
\mathbf{P}(B \text { is deviant }) \leq \frac{\operatorname{Var}\left(S_{n}\right)}{\left(\frac{1}{2} \mathbf{E}\left(S_{n}\right)\right)^{2}}=\frac{\sum_{i=1}^{n} \gamma_{i}^{2}}{\left(\sum_{i=1}^{n} \gamma_{i}\right)^{2}} \leq C_{2}\left(\sum_{i=1}^{n} \gamma_{i}\right)^{-1},
$$

since $\gamma_{i}^{2} \leq C_{2} \gamma_{i}$ by (3.4). Denoting by $\mathcal{D}_{n}$ the collection of deviant squares in $\mathcal{G}_{k_{n}}$ and using independence of $B$ being deviant from the event $\left(B \in \mathcal{F}_{k_{n}}\right)$, we obtain that

$$
\#\left(\mathcal{F}_{k_{n}} \cap \mathcal{D}_{n}\right) \leq C_{2} \cdot \# \mathcal{F}_{k_{n}}\left(\sum_{i=1}^{n} \gamma_{i}\right)^{-1} .
$$

By the definition of measure $\mu$ we have

$$
\mu\left(\mathcal{R} \backslash \bigcup_{B \in \mathcal{D}_{n}} B\right) \geq 1-C_{2}\left(\sum_{i=1}^{n} \gamma_{i}\right)^{-1}
$$

Note that $\alpha_{i}=j$ for $k_{j} \leq i<k_{j+1}$, hence denoting $k_{0}=0$ we have

$$
\sum_{i=0}^{k_{n}-1} \lambda_{i}=\sum_{j=0}^{n-1} \sum_{i=k_{j}}^{k_{j+1}-1} \lambda_{i}=\sum_{j=0}^{n-1} 4^{-j} \sum_{i=k_{j}}^{k_{j+1}-1} 2^{i}<\sum_{j=0}^{n-1} 4^{-j} 2^{k_{j+1}}=4 \sum_{j=1}^{n} \gamma_{j} .
$$

Observe that $k_{n} \rightarrow \infty$, otherwise $\mathcal{R}$ is a finite set. Thus, $\sum_{i=1}^{n} \gamma_{i} \rightarrow \infty$ as $n \rightarrow \infty$ by (3.2), and we can find $n(j) \in \mathbb{N}, j \geq 1$, such that

$$
C_{2}^{-1} \sum_{i=1}^{n(j)} \gamma_{i}>2^{j+1}
$$

Let

$$
\mathcal{R}^{\prime}:=\mathcal{R} \backslash \bigcup_{j=1}^{\infty} \bigcup_{B \in \mathcal{D}_{n(j)}} B
$$

We have $\mu\left(\mathcal{R}^{\prime}\right) \geq 1-\frac{1}{2} \sum_{j=1}^{\infty} 2^{-j}=\frac{1}{2}$ by (3.5) and (3.6). Thus, $\left.\mu\right|_{\mathcal{R}^{\prime}}$ is a positive measure, and $\mu\left(B_{r} \cap \mathcal{R}^{\prime}\right) \leq \mu\left(B_{r}\right) \leq C_{1} \cdot \varphi(r)$ for any ball $B_{r}$ of radius $r$, hence $\mathcal{H}^{\varphi}\left(\mathcal{R}^{\prime}\right)>0$ by the Mass Distribution Principle.

\section{Proof of Proposition 2.1 and Theorem 1.1}

Denote

$$
\mathcal{R}_{k_{n}}^{\prime}=\mathcal{R}_{k_{n}} \backslash \bigcup_{B \in \mathcal{D}_{n}} B
$$

Lemma 4.1. Let $\ell$ be a line intersecting $[0,1]^{2}$ that does not hit any vertices of the squares in $\mathcal{G}_{k_{n}}$. Then

$$
\mathbf{P}\left(\mathcal{R}_{k_{n}}^{\prime} \cap \ell \neq \emptyset\right) \leq 64 \cdot\left(\sum_{i=1}^{n} \gamma_{i}\right)^{-1}
$$


Proof of Theorem 1.1 assuming Lemma 4.1. Observe that $\mathcal{R}^{\prime}=\bigcap_{j=1}^{\infty} \mathcal{R}_{k_{n(j)}}^{\prime}$, hence Lemma 4.1 implies that the probability of $\ell$ hitting $\mathcal{R}^{\prime}$ equals zero. Let $\theta \in[0, \pi]$ be such that the line $y \cos \theta=x \sin \theta$ is orthogonal to $\ell$, and let $\mathbf{n}$ be the unit normal vector for $\ell$. Then by Fubini's Theorem,

$$
\mathbf{E}\left[\mathcal{L}^{1}\left(p_{\theta}\left(\mathcal{R}^{\prime}\right)\right)\right]=\int_{\mathbb{R}} \mathbf{P}\left(\mathcal{R}^{\prime} \cap(\ell+t \mathbf{n}) \neq \emptyset\right) d t=0 .
$$

The proposition, and hence the theorem, follow by integrating over $\theta$.

Proof of Lemma 4.1. Let $\alpha$ be the angle that $\ell$ forms with the horizontal. First we assume that $\alpha \in[0, \pi / 2]$ and then indicate how to consider the case $\alpha \in(\pi / 2, \pi]$. Let

$$
A_{n}(\ell)=\#\left\{B \in \mathcal{G}_{k_{n}}: B \cap \ell \neq \emptyset\right\} .
$$

Observe that

$$
A_{n}(\ell) \leq 2^{k_{n}+1} .
$$

To verify this we may assume, using symmetry, that $\ell$ forms an angle in $[0, \pi / 4]$ with the horizontal. Then $\ell$ intersects at most two squares in each of the $2^{k_{n}}$ columns of $\mathcal{G}_{k_{n}}$, and (4.1) follows.

Recall that none of the squares in $\mathcal{R}_{k_{n}}^{\prime}$ are deviant, i.e.

$$
B \in \mathcal{R}_{k_{n}}^{\prime} \Rightarrow(1 / 4) \sum_{i=1}^{n} \gamma_{i} \leq S_{n}(B) \leq(3 / 4) \sum_{i=1}^{n} \gamma_{i},
$$

where $S_{n}(B)=\sum\left\{\gamma_{i}: i \leq n, \omega_{k_{i}}(B) \in\{0,2\}\right\}$.

(i) Say that $B \in \mathcal{G}_{2 n}$ is 0-rich if

$$
\sum\left\{\gamma_{i}: i \leq n, \omega_{k_{i}}(B)=0\right\} \geq(1 / 8) \sum_{i=1}^{n} \gamma_{i} .
$$

(ii) Say that $B \in \mathcal{G}_{2 n}$ is 2 -rich if

$$
\sum\left\{\gamma_{i}: i \leq n, \omega_{k_{i}}(B)=2\right\} \geq(1 / 8) \sum_{i=1}^{n} \gamma_{i} .
$$

By the definition of deviant squares, every non-deviant square is either 0-rich or 2-rich (or both). Consider the events

$$
Z_{i}=\left\{\exists B \in \mathcal{F}_{k_{n}}: B \text { is } i \text {-rich \& } B \cap \ell \neq \emptyset\right\} \text { for } i=0,2 .
$$

Since $\mathcal{R}_{k_{n}}^{\prime}$ contains only non-deviant squares from $\mathcal{F}_{k_{n}}$, we have

$$
\mathbf{P}\left(\mathcal{R}_{k_{n}}^{\prime} \cap \ell \neq \emptyset\right) \leq \mathbf{P}\left(Z_{0}\right)+\mathbf{P}\left(Z_{2}\right) \text {. }
$$

Let us estimate $\mathbf{P}\left(Z_{0}\right)$. We have

$$
\mathbf{E}\left[\#\left\{B \in \mathcal{F}_{k_{n}}: B \cap \ell \neq \emptyset\right\} \mid Z_{0}\right] \leq \frac{\mathbf{E}\left[\#\left\{B \in \mathcal{F}_{k_{n}}: B \cap \ell \neq \emptyset\right\}\right]}{\mathbf{P}\left(Z_{0}\right)} .
$$

Observe that

$$
\mathbf{P}\left(B \in \mathcal{F}_{k_{n}}\right)=\# \mathcal{F}_{k_{n}} / \# \mathcal{G}_{k_{n}}=4^{n} / 2^{2 k_{n}}=4^{n-k_{n}}
$$

for any $B \in \mathcal{G}_{k_{n}}$. Writing

$$
\#\left\{B \in \mathcal{F}_{k_{n}}: B \cap \ell \neq \emptyset\right\}=\sum_{B \in \mathcal{G}_{k_{n}}} \mathbf{1}_{\left\{B \in \mathcal{F}_{k_{n}}: B \cap \ell \neq \emptyset\right\}}
$$


and using (4.3) we obtain by (4.1) that

$$
\mathbf{E}\left[\#\left\{B \in \mathcal{F}_{k_{n}}: B \cap \ell \neq \emptyset\right\}\right]=A_{n}(\ell) \cdot 4^{n-k_{n}} \leq 2 \cdot 2^{2 n-k_{n}} .
$$

Thus by (4.2),

$$
\mathbf{P}\left(Z_{0}\right) \leq \frac{2^{2 n-k_{n}+1}}{\mathbf{E}\left[\#\left\{B \in \mathcal{F}_{k_{n}}: B \cap \ell \neq \emptyset\right\} \mid Z_{0}\right]} .
$$

It remains to estimate the denominator in (4.5) from below. Let

$$
\Psi_{0}:=\left\{Q \in \mathcal{G}_{k_{n}}: Q \text { is } 0 \text {-rich \& } Q \cap \ell \neq \emptyset\right\} .
$$

Order the squares in $\mathcal{G}_{k_{n}}$ hit by $\ell$ from left to right and from bottom to top. This is a total order by the assumption on the slope of the line $\ell$. For $Q \in \Psi_{0}$ consider the event

$$
Y_{Q}=\left\{Q \text { is the first square in } \Psi_{0} \text { such that } Q \in \mathcal{F}_{k_{n}}\right\} .
$$

Then $Z_{0}=\bigcup_{Q \in \Psi_{0}} Y_{Q}$ is a disjoint union, and so, for any random variable $f$,

$$
\mathbf{E}\left[f \mid Z_{0}\right]=\sum_{Q \in \Psi_{0}} \frac{\mathbf{P}\left(Y_{Q}\right)}{\mathbf{P}\left(Z_{0}\right)} \mathbf{E}\left[f \mid Y_{Q}\right] \geq \min _{Q \in \Psi_{0}} \mathbf{E}\left[f \mid Y_{Q}\right] .
$$

Fix $Q \in \Psi_{0}$. We have

$$
\mathbf{E}\left[\#\left\{B \in \mathcal{F}_{k_{n}}: B \cap \ell \neq \emptyset\right\} \mid Y_{Q}\right]=\sum_{B \in \mathcal{G}_{k_{n}}: B \cap \ell \neq \emptyset} \mathbf{P}\left(B \in \mathcal{F}_{k_{n}} \mid Y_{Q}\right) .
$$

Fix $i$ such that $\omega_{k_{i}}(Q)=0$, and denote by $\widetilde{Q}$ the dyadic square in $\mathcal{G}_{k_{i}-1}$ having the address $\omega(\widetilde{Q})=\omega_{1}(Q) \ldots w_{k_{i}-1}(Q)$. The fact that $Q \in \mathcal{F}_{k_{n}}$ implies that $\widetilde{Q} \in \mathcal{F}_{k_{i}-1}$. (Note that by definition, $[0,1]^{2} \supset \mathcal{R}_{k_{1}} \supset \ldots \supset \mathcal{R}_{k_{n}}$.) Recall that the step $k_{i}$ is deterministic, so all four offspring of $\widetilde{Q}$ are in $\mathcal{F}_{k_{i}}$. Since the slope of $\ell$ is positive, $\ell$ intersects at least $\frac{1}{2} 2^{k_{n}-k_{i}}$ squares $B \in \mathcal{G}_{k_{n}}$ whose addresses start with $\omega(\widetilde{Q}) k$, for $k \in\{1,2,3\}$ (see Figure 1). For each of these squares we have (using the independence of $Y_{Q}$ from the random choices involving the descendants of $\omega(\widetilde{Q}) k$ with $k \in\{1,2,3\})$ that

$$
\mathbf{P}\left(B \in \mathcal{F}_{k_{n}} \mid Y_{Q}\right)=\mathbf{P}\left(B \in \mathcal{F}_{k_{n}} \mid \widetilde{Q} \in \mathcal{F}_{k_{i}-1}\right)=4^{k_{i}-k_{n}+n-i} .
$$

This is because we made $(n-i)$ deterministic steps between stages $k_{i}$ and $k_{n}$, and hence $\left(k_{n}-k_{i}\right)-(n-i)$ random steps. Therefore, the sum of $\mathbf{P}\left(B \in \mathcal{F}_{k_{n}} \mid Y_{Q}\right)$ over the set of squares

$$
\mathcal{U}_{i}=\left\{B \in \mathcal{G}_{k_{n}},: B \cap \ell \neq \emptyset, \quad\left\{\omega_{j}(B)\right\}_{1}^{k_{i}-1}=\left\{\omega_{j}(Q)\right\}_{1}^{k_{i}-1}, \quad \omega_{k_{i}}(B) \in\{1,2,3\}\right\},
$$

is at least $\frac{1}{2} 2^{k_{n}-k_{i}} \cdot 4^{k_{i}-k_{n}+n-i}=\frac{1}{2} 2^{2 n-k_{n}} \gamma_{i}$. Note that the sets $\mathcal{U}_{i}$ are disjoint for distinct $i$ with $\omega_{k_{i}}(Q)=0$. Thus, by the definition of 0-rich squares, the right-hand side of (4.7) is at least

$$
(1 / 2) 2^{2 n-k_{n}} \cdot \sum\left\{\gamma_{i}: i \leq n, \omega_{k_{i}}(Q)=0\right\} \geq(1 / 16) 2^{2 n-k_{n}} \cdot \sum_{i=1}^{n} \gamma_{i} .
$$

Therefore by (4.6),

$$
\mathbf{E}\left[\#\left\{B \in \mathcal{F}_{k_{n}}: B \cap \ell \neq \emptyset\right\} \mid Z_{0}\right] \geq 2^{2 n-k_{n}-4} \cdot \sum_{i=1}^{n} \gamma_{i}
$$


Together with (4.5), this yields that

$$
\mathbf{P}\left(Z_{0}\right) \leq 32 \cdot\left(\sum_{i=1}^{n} \gamma_{i}\right)^{-1} .
$$

The estimate $P\left(Z_{2}\right) \leq 32 \cdot\left(\sum_{i=1}^{n} \gamma_{i}\right)^{-1}$ is similarly proved. We consider the set $\Psi_{2}$ of squares in $\mathcal{G}_{k_{n}}$ hit by $\ell$ that are 2-rich and condition on the last square in $\Psi_{2}$ which belongs to $\mathcal{F}_{k_{n}}$.

This concludes the proof of the lemma when the angle $\alpha$ is in $[0, \pi / 2]$. In the case when $\alpha \in[\pi / 2, \pi]$ we interchange the roles of the subsquares $\{0,2\}$ and $\{1,3\}$ and use that for a non-deviant square $B$ we have

$$
\sum\left\{\gamma_{i}: i \leq n, \omega_{k_{i}}(B) \in\{1,3\}\right\} \geq(1 / 4) \sum_{i=1}^{n} \gamma_{i}
$$

\section{Proof of Lemma 1.2}

Let

$$
\varphi_{1}(s)=\inf _{r}\left\{(s / r)^{2} \varphi(r): r \leq s\right\} .
$$

Then $\varphi_{1}(s) \leq \varphi(s)$, so the left-hand side in (1.3) is clear. For the right-hand side consider a cover of a set $A$ for $\mathcal{H}^{\varphi_{1}}$. Let $S$ be a set in this cover where $S$ has diameter $s$. Then we can find $r \leq s$ with $(s / r)^{2} \varphi(r)<2 \varphi_{1}(s)$. Let $v<r$ be the largest number of the form $s / 2^{k}$. Then $(s / v)^{2} \varphi(v)<8 \varphi_{1}(s)$. Now $S$ may be covered by a square of side $s$, hence by $4^{k+1}=4(s / v)^{2}$ squares of side $v / 2$. Replacing $S$ by these squares and using them in the Hausdorff sum for $\mathcal{H}^{\varphi}$ we conclude that $\mathcal{H}^{\varphi}(A) \leq 32 \mathcal{H}^{\varphi_{1}}(A)$.

The condition $\varphi_{1}(s) / s^{2} \downarrow$ is immediate, since $\varphi_{1}(s) / s^{2}=\inf \left\{\varphi(r) / r^{2}: r \leq s\right\}$. It remains to check that $\varphi_{1}(s) \uparrow$. Suppose $s<t$. Then $\varphi_{1}(t)=\min \left\{A_{1}, A_{2}\right\}$, where

$$
A_{1}=\inf _{r \leq s}\left\{\left(t^{2} / r^{2}\right) \cdot \varphi(r)\right\} \text { and } A_{2}=\inf _{r \in[s, t]}\left\{\left(t^{2} / r^{2}\right) \cdot \varphi(r)\right\} .
$$

It is clear that $A_{1} \geq \varphi_{1}(s)$, and $A_{2} \geq\left(t^{2} / r^{2}\right) \cdot \varphi(s) \geq \varphi(s) \geq \varphi_{1}(s)$ since $\varphi(r)$ is increasing. The lemma is proved.

\section{Proof of Corollary 1.3}

We only need to prove that (iii) $\Rightarrow$ (i). We prove the contrapositive, i.e., given $\psi$ satisfying the regularity conditions, we show that $\mathcal{H}^{\psi}$ is not absolutely continuous to $\mathcal{I}^{m}$ as Borel measures on $\mathbb{R}^{n}$.

Let $\varphi(r)=\psi(r) / r^{m-1}$. Then Proposition 2.1 yields a Borel set $A \subset \mathbb{R}^{2}$ such that $\mathcal{H}^{\varphi}(A)>0$ and $\mathcal{I}^{1}(A)=0$. Define $B \subset \mathbb{R}^{n}$ as the Cartesian product $B=$ $A \times[0,1]^{m-1} \times\{(0, \ldots, 0)\}$ (appending $n-m-1$ zeros). Then it is standard to verify that $\mathcal{H}^{\psi}(B)>0$ yet $\mathcal{I}^{m}(B)=0$. For the latter, observe that for a generic $m$-dimensional subspace $V$ and $a \in V$, the $(n-m)$-dimensional subspace $p_{V}^{-1}(a)$ intersected with $\mathbb{R}^{m+1} \times\{(0, \ldots, 0)\}$, is a line which hits $B$ if and only if its projection on the first two coordinates hits $A$. Thus the inner integral in (1.4) is zero for a generic $V$. 


\section{REFERENCES}

1. L. Carleson, Selected problems on exceptional sets, Van Nostrand, Princeton (1967). MR0225986 (37:1576)

2. H. Joyce and P. Mörters, A set with finite curvature and projections of zero length, J. Math. Anal. Appl. 247 (2000), no. 1, 126-135. MR.1766928 (2001j:28006)

3. R. Kaufman, On Hausdorff dimension of projections, Mathematika 15 (1968), 153-155. MR0248779(40:2030)

4. J. Marstrand, Some fundamental geometrical properties of plane sets of fractional dimension, Proc. London Math. Soc. 4 (1954), 257-302. MR0063439 (16:121g)

5. P. Mattila, Geometry of Sets and Measures in Euclidean Spaces, Cambridge University Press, 1995. MR333890 (96h:28006)

6. P. Mattila, Hausdorff dimension, projections, and the Fourier transform, Publ. Math. 48 (2004), 3-48. MR2044636 (2004k:28018)

7. Y. Peres and B. Solomyak, How likely is Buffon's needle to fall near a planar Cantor set, Pacific J. Math. 204 (2002), 473-496. MR.1907902(2003h:28010)

Department of Statistics, University of California, Berkeley, California 94720

E-mail address: peres@stat.berkeley.edu

Department of Mathematics, University of Washington, Box 354350, Seattle, WashINGTON 98195

E-mail address: solomyak@math.washington.edu 